\title{
ACTIVITY OF REDOX ENZYMES IN SYMBIOTIC SYSTEMS OF SOYBEAN- BRADYRHIZOBIUM JAPONICUM UNDER THE ACTION OF EXOGENOUS LECTIN AND DIFFERENT WATER SUPPLY
}

\section{S. Y. Kots, L. I. Rybachenko, L. M. Mykhalkiv}

Symbiotic interaction between leguminous plants and nodule bacteria is a complex physiological process that is regulated by a signalling system between macro- and microsymbionts. Invasion of rhizobia into legume cells of root fibrils as well as pathogenesis cause intensification of oxidative processes in plant cells, accompanied by increased content of reactive oxygen species that induce a series of reactions in the plant organism. In turn, their number is controlled by a multilevel antioxidant protection system.

It is shown that peroxidases, including ascorbate- and guaiacolperoxidase, play an important role in the processes of nodule formation and functioning of symbiotic apparatus. Zhyznevska et al. showed that during formation of symbiotic systems, cellular cytosol of effective lupine nodules shows blockade of synthesis of the range of proteins, however, new proteins - nodulins — are synthesized, and peroxidase reaction is considered as a response to the penetration of rhizobia into the plant cell. Also, there is an assumption that the role of these enzymes may involve maintenance of stability of leghemoglobin structure. This group of authors noted an important role of catalase in the formation and functioning of symbiotic systems. It was established that the activity of this enzyme correlated with activity of symbiotic nitrogen fixation in root nodules of legumes.

Plant protection system in the conditions of oxidative stress has a multistage nature and is not limited to the functioning of redox enzymes. There is an assumption that the components of forming nonspecific defence reactions of plants may be plant lectins as well. It is known know that they are involved in intercellular recognition of phytopathogens by plants involved in the formation of non-specific adaptive syndrome at low temperature, in the formation of protective plant organism response to the action of hypo- and hyperthermia, heavy metals, UV radiation, water, osmotic and salt stress.

The aim of our work was to investigate the activity of ascorbate-, guaiacolperoxidase and catalase in soybean plants under the influence of exogenous lectin and different water supply that will reveal additional aspects of the impact of the protein on the functioning of symbiotic systems under stress.

Materials and methods. In this work, we used plants of soybean (Glycine max (L.) Merr.) Vasylkivska variety and an active strain of rhizobia Bradyrhizobium japonicum 634b.

Before sowing, seeds were sterilized by $70 \%$ ethanol solution and washed with running water. One part was incubated with a solution of commercial soybean seed lectin (Lviv, "Lektynotest") at a concentration of $100 \mu \mathrm{g} / \mathrm{mL}$, then inoculated with a bacterial suspension. The rest of the seeds were incubated using water, and inoculated rhizobia suspension that was pre-incubated with lectin. The 
duration of incubation with lectin was 20 hours, and rhizobia inoculation -1 hour. Thus, it was examined two ways of using lectin: seed treatment and rhizobia treatment. A variant with inoculation of plants with rhizobia without using lectin was used as a control.

Plants were grown in 16-kilogram Wagner vessels in sand culture with the introduction of nutritional Hellriegel mixture with 0.25 normal range of nitrogen under natural lighting, optimal (60\% of complete humidity rate (CHR)) and insufficient (30\% of CHR) water supply. Drought was created by a controlled irrigation for two weeks, starting with phase of three true leaves, then irrigation was re-initiated to $60 \% \mathrm{CHR}$.

Activity of guaiacolperoxidase (GP) was determined by the method of Egley G. H., ascorbateperoxidase - by the method of Verma S. and Dubey R. S., catalase — by the method of Doliba I. M.

The content of total soluble protein in the enzymatic extract was determined by Bradford M. M. Measurement of enzymatic activity and protein content was performed in 3x biological replications. Obtained data were statistically processed using Microsoft Excel.

Therefore, the analysis of the results of our studies showed changes in activity of antioxidative enzymes important in the formation and functioning of symbiosis, namely ascorbate-, guaiacolperoxidase and catalase, under the influence of exogenous lectin. It should be noted that the maximum increase in activity of all studied enzymes in the roots of soybeans, which is exposed to drought, was shown in the variant with the use of this protein as a component of inoculation suspension. This effect from the use of such protein can confirm its importance in the regulation of the formation and functioning of symbiosis and in reactions of inoculated soybean plants to the influence of drought. 